\title{
Evaluation of the effect of camel milk in comparison with standard medicinal therapy on asthma patients: a parallel clinical trial
}

Mohammad Ravaghi ${ }^{1}$, Mahdi Yousefi ${ }^{2}$, Davood Attaran ${ }^{3}$, Saeed Zibaee ${ }^{4}$, Roshanak Salari ${ }^{5}$, Malihe Dadgar Moghaddam $^{6}$, Farahzad Jabbari Azad ${ }^{7}$, Seyed Mousalreza Hosseini ${ }^{8}$

${ }^{1}$ Ph.D. Candidate of Persian Medicine, Student Research Committee, School of Persian and Complementary Medicine, Mashhad University of Medical Sciences, Mashhad, Iran

${ }^{2}$ Ph.D. of Persian Medicine, Assistant Professor, Department of Persian Medicine, School of Persian and Complementary Medicine, Mashhad University of Medical Sciences, Mashhad, Iran

${ }^{3}$ M.D., Pulmonologist, Professor, Department of Pulmonology, School of Medicine, Mashhad University of Medical Sciences, Mashhad, Iran

${ }^{4}$ Ph.D. of Microbiology, Associate Professor, Department of Veterinary Research and Biotechnology, Razi Vaccine and Serum Research Institute of Mashhad, Mashhad, Iran

${ }^{5}$ Ph.D. of Drug Control, Assistant Professor, Department of Pharmaceutical Sciences in Persian Medicine, School of Persian and Complementary Medicine, Mashhad University of Medical Sciences, Mashhad, Iran

${ }^{6}$ M.Sc. of Community Medicine, Assistant Professor, Department of Community Medicine, Faculty of Medicine Mashhad University of Medical Sciences, Mashhad, Iran

${ }^{7}$ M.D., Allergy and Clinical Immunologist, Professor, Head of Allergy Research Center, Department of Immunology and Allergy, Mashhad University of Medical Sciences, Mashhad, Iran

${ }^{8}$ M.D., Gasteroenterologist, Associate Professor, School of Medicine, Mashhad University of Medical Sciences, Mashhad, Iran

\section{Type of article: Original}

\begin{abstract}
Background: Asthma is one of the most common chronic diseases in the world by which more than 300 million people are affected. In conventional medicine for asthma treatment, more emphasis is on drug therapy, which has complications and contraindications as well as high costs, so we are investigating to identify the effect of camel's milk on the symptoms of patients with asthma.

Objective: To examine the effects of camel milk in comparison with standard medicinal therapy on asthma patients.

Methods: This randomized clinical trial was conducted on 46 patients with asthma. The study was conducted in Ghaem Hospital lung clinic in Mashhad, Iran, from May 2016 to November 2017. The participants were randomly divided into control and intervention groups (n, 23 per group) and were assessed both pretreatment and post treatment (before, and three months after treatment). Patients with asthma based on clinical and spirometric criteria were included in the study and divided into two groups of control and intervention. Twenty-three patients with asthma were entered into each group. The control group received routine treatments (steroid and $\beta$-agonist inhalation) and the intervention group, which received the usual treatments, plus pasteurized camel milk. Camel milk was administered two times a day ( 8 am and $8 \mathrm{pm} 250 \mathrm{ml}$ without additives). Data were collected using a chronic obstructive pulmonary disease (COPD) assessment test (CAT) questionnaire score and spirometry. The results were analyzed and compared in SPSS version 11.5, using paired t-test, Chi-Square test, and independent sample t-test. The significance level was set at 0.05 .
\end{abstract}

\section{Corresponding author:}

Assistant Professor Dr. Seyed Mousalreza Hosseini, School of Medicine, Mashhad University of Medical Sciences, Mashhad, Iran. Tel: +98.9151039519, Email: hoseinimr@mums.ac.ir

Received: July 07, 2018, Accepted: September 27, 2018, Published: March 2019

iThenticate screening: February 03, 2019, English editing: February 08, 2019, Quality control: February 12, 2019

This article has been reviewed / commented by four experts

Funding / research project approval: Mashhad University of Medical Sciences (Ref: 941287)

Ethics approval: Ethics Committee for Research at Mashhad University of Medical Sciences (Ref: 941287, dated May 28, 2016).

(C) 2019 The Authors. This is an open access article under the terms of the Creative Commons Attribution-NonCommercialNoDerivs License, which permits use and distribution in any medium, provided the original work is properly cited, the use is non-commercial and no modifications or adaptations are made. 
Results: In this study, changes in the mean of forced expiratory volume during one second (FEV1) measurement and FEV1 percent and CAT questionnaire score in both groups, before and after treatment, were significant. But the mean of FEV1 (measurement and percent) in the intervention group was significantly higher than the control group. The mean of FEV1 percent and FEV1 measurement in the intervention and the control groups were $\mathrm{p}<0.001, \mathrm{p}=0.002, \mathrm{p}=0.001$ and $\mathrm{p}=0.049$ respectively, while the mean of CAT questionnaire scores were not significantly different between two groups $(\mathrm{p}<0.001, \mathrm{p}<0.001$ respectively). In addition, the mean of FEV1 percent and CAT questionnaire score difference in the intervention group were significantly higher than in the control group ( $p=0.001, p<0.001$ respectively), but the mean of FEV1 measurement differences between the intervention and the control group were not significant $(\mathrm{p}=0.05)$.

Conclusion: In our study, we found that camel milk, with the standard asthma treatment, would be very helpful. However, there is a need for further studies with a larger sample size on the effect of this nutrient.

Clinical Trial Registration: This study was registered at the Iranian Registry of Clinical Trials (http://www.irct.ir) with the registration code IRCT2016102930541N1.

Funding: This study was supported by Mashhad University of Medical Sciences Research Council, Mashhad, Iran (Ref: 941287). The authors have no conflicts of interest.

Keywords: Asthma, Camel milk, Medicinal therapy

\section{Introduction}

Asthma is one of the most common chronic diseases in the world by which more than 300 million people are affected (1). In Iran, the prevalence of asthma was $4.56 \%$ and $4.17 \%$ among males and females in 2015 , respectively and the prevalence of asthma resistant to treatment was about 2.8\%-3.8\% (2, 3). In a 2007-2010 cross sectional study by Sharifi L et al. in Iran, the total cost of asthma was 590.22 \pm 32.18 USD. They demonstrated that compared to the patient's family income, this figure is significant (4). In conventional medicine, asthma treatment consists of two groups of drugs, bronchodilators and inflammation controllers; Bronchodilators quickly improve the disease symptoms, and the controllers are inflammation eliminators. Bronchodilators include $\beta 2$-agonists, anticholinergics, and theophylline, and controllers include inhalation and edible corticosteroids, anti-leukotrienes, anti-IgE antibodies (5). Asthma drug regimens, especially in high-dose and long-term use may lead to drug side effects (6). Currently, the World Health Organization (WHO) suggests traditional treatments in underdeveloped countries (7). Complementary and alternative medicine (CAM) is defined as "A group of diverse medical and health care systems, practices, and products that are not generally considered to be part of conventional medicine" and there is a rising tendency, around the world, to take advantage of CAM $(8,9)$. Traditionally, camel milk has been used for asthma treatment in Asia and Africa (10). There are currently 171,500 camels scattered throughout most provinces of Iran, which provide appropriative dairy sources (11). Camel milk is believed to modulate the immune system. In some inflammatory diseases, such as hepatitis, allergies, lactose intolerance and liver damage induced by alcohol, the benefits of camel milk can be related to important immunological components such as lysozyme, lactoferrin, lactoperoxidase, etc. Besides this, antibodies of camel milk have specific structures and functions, which may result in therapeutic benefits with the mechanism of immune system regulation in inflammatory diseases (12). A study showed its ability to ameliorate severe food allergies in children in a short time with a long time interval (13). It is used extensively within a variety of societies for its proposed healing features and disease prevention (14). Some of the more common indications to using it include diabetes, allergies, immune disorders, various infections, heavy metal toxicity, colitis, and alcohol-induced toxicity and cancer (15-19). So far, no study has been done on the efficacy of camel milk in patients with asthma; only Boskabadi et al. showed the effects of camel milk on dilation of trachea isolated from guinea pigs, in which the bronchodilatory effects were specified (20). According to recent studies on the effect of camel's milk on animal samples, and also on the unique properties of camel's milk, and on the other hand, the numerous complications and contraindications of drugs used in asthma along with high costs, we decided to evaluate the effects of camel milk on patients with asthma. The main objective of this study was to evaluate the effectiveness of camel milk on asthmatic patients compared with standard medicinal therapy, and its specific objectives were to evaluate the effectiveness of standard medicinal therapy in the treatment of asthmatic patients based on the CAT questionnaire and spirometry, evaluate the effectiveness of camel milk in the treatment of asthmatic patients based on the CAT questionnaire and spirometry, and finally compare the effectiveness of camel milk with conventional therapy for the treatment of asthmatic patients based on the CAT questionnaire score and spirometry.

\section{Material and Methods}

\subsection{Trial design}

This parallel, randomized clinical trial study was conducted in Ghaem Hospital lung clinic in Mashhad, Iran, from May 2016 to November 2017 on asthmatic patients referring to this specialized center. 


\subsection{Participants}

The participants under study were people with asthma confirmed by pulmonologist (based on asthma typical symptoms and positive spirometry), referring to the specialized lung center of Ghaem Hospital, affiliated to Mashhad University of Medical Sciences in Mashhad, Iran. They were randomly divided into two groups of control and intervention ( $\mathrm{n}=23$ per group) and were assessed both pretreatment and post treatment (before, and three months after treatment). The control group received routine treatments (steroid and $\beta$-agonist inhalation) and the intervention group received the usual treatments, plus pasteurized camel milk. Camel milk was administered two times a day (8 am and $8 \mathrm{pm} 250 \mathrm{ml}$ without additives) (Figure 1). The spirometer device used for patients was CHEST HI-801 made in Japan.

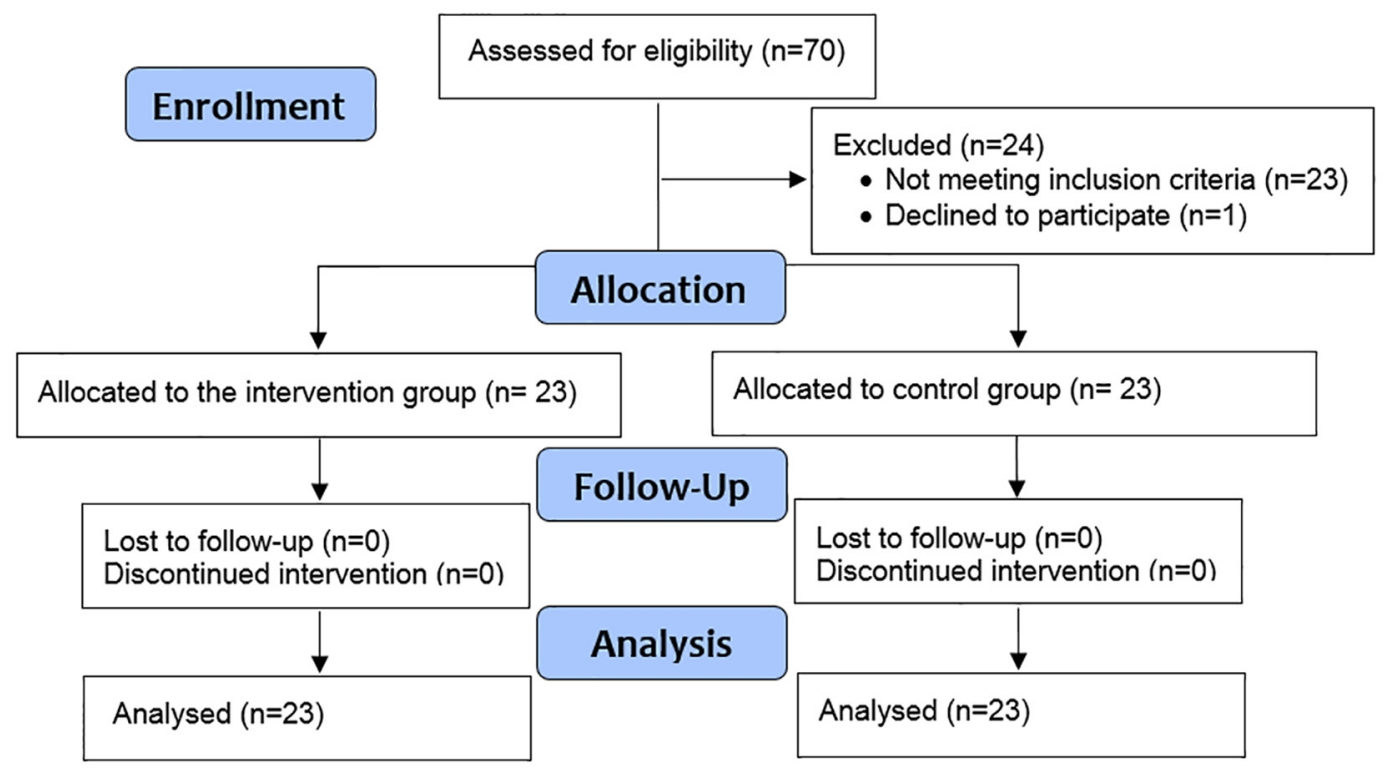

Figure 1. CONSORT 2010 flowchart of the study

\subsection{Inclusion criteria}

Patients aged 15-50 years old with asthma diagnosis referred by pulmonologist were included in this study. Asthma diagnosis was done by positive history of asthma symptoms such as cough, shortness of breath, wheeze and chest tightness, which were confirmed by FEV1/FVC ratio less than 0.80 at least one time when FEV1 is less than 0.70 or increased FEV1 by more than $12 \%$ and $200 \mathrm{ml}$ after inhaling a bronchodilator in spirometry (5).

\subsection{Exclusion criteria}

Exclusion criteria included history of camel milk consumption in the past, sensitivity to the camel milk, chronic diseases, and hospitalization during treatment, admittance to the emergency department, pregnancy, breast-feeding and cigarette smokers.

\subsection{Sample size}

The sample size, considering that there has not been a similar study on the effect of camel milk on asthmatics patients, was based on an unpublished author's pilot study with $95 \%$ success rate in treatment, and the median outcome success of $60 \%$ based on FEV1 was forty-six cases (21). The required sample size was $n=23$.

\subsection{Randomization and blinding}

Patients who had the criteria for entering the study were randomly assigned to control and intervention groups. Randomization was done using a random numbers table but blinding was not done in the study. Patients were unaware of the treatment received by other participants in the study, and the analyst was not aware of the results of the patients.

\subsection{Outcome}

Age, sex, FEV1 measurement, FEV1 and FEV1/FVC percent, (spirometric indices) and CAT questionnaire score were the variables of this study. 


\subsection{Intervention and measurements}

The patients in the control group received routine asthma treatments (steroid and $\beta$-agonist inhalation) without placebo. In addition to the usual treatments, pasteurized camel milk (raw milk that came from Golestan province and was pasteurized by Razi Research Institute) was administered two times a day (8 am and $8 \mathrm{pm} 250 \mathrm{ml}$ without additives) for the patients in the intervention group. None of the patients in the control or intervention group had a history of camel milk consumption in the past. During each treatment period, the patients were visited and their clinical symptoms were evaluated and recorded before and after the three-month treatment period. Complications associated with the use of each drug were recorded. Before and after the intervention, spirometry indices, forced expiratory volume in the first second (FEV1), FEV1/FVC percent and chronic obstructive pulmonary disease assessment test (CAT) questionnaire score were evaluated and the results were compared using statistical tests.

\subsection{Statistical Analyses}

Data were analyzed by SPSS version 11.5 (SPSS Inc., Chicago, Illinois, USA). Quantitative and qualitative data were reported regarding mean and standard deviation and number and percentage in tables and charts, respectively. For data comparisons before and after intervention in each group, in case of normal distribution of data, the parametric paired t-test was used. For comparison between quantitative variables, Chi-Square test was used and for qualitative variables, independent sample t-test was used. All tests were two-sided with a significance level of $\mathrm{p}<0.05$.

\subsection{Research ethics}

Declaration of Helsinki - ethical principles for medical research involving human subjects (adopted by the 18th General Assembly of World Medical Association [WMA], Helsinki, Finland, June 1964 and last amended by the 64th WMA General Assembly, Fortaleza, Brazil, October 2013) was considered throughout the designing of the study protocol and its implementation. The present study has been approved by the Ethics Committee for Research at Mashhad University of Medical Sciences (Ref: 941287, dated May 28, 2016). This study was registered at the Iranian Registry of Clinical Trials (http://www.irct.ir) with the registration code IRCT2016102930541N1. Patients were informed about this study and the consent forms were signed individually. All patients entered voluntarily into the study and they were allowed to exit the study at any time. In order to ensure that no side effects or complications occurred to the participants, an accessible telephone number was provided for contact at any time if medical consultation was required. All information of the participants was confidential.

\section{Results}

In this study, the sample size of patients was calculated as 46 (23 patients in each group). Of these, in each group, 10 $(43.5 \%)$ patients were male and $13(56.5 \%)$ patients were female. So there was no significant difference in distribution of gender between the two groups $(\mathrm{p}=0.99)$. The mean age in the intervention group was $44.21 \pm 5.93$ years and in the control group was $41.00 \pm 6.94$ years, which was not statistically significant between the two groups. Therefore, there was no significant difference between the two groups in terms of age $(p=0.098)$. The mean of FEV1 measurement, FEV1 percent, FEV1/FVC percent and CAT questionnaire score had no significant differences between intervention and control groups before study ( $\mathrm{p}=0.440,0.207,0.098$ and 0.365 respectively). In this study, changes in the mean of FEV1 (measurement and percent) and CAT questionnaire in both groups, before and after treatment, were significant. But the mean of FEV1 (measurement and percent) in the intervention group was significantly higher than the control group ( $<<0.001, p=0.002$ vs. $p=0.001$ and $p=0.049$ respectively), while the mean of CAT questionnaire score was not significantly different between the two groups $(\mathrm{p}<0.001, \mathrm{p}<0.001$ respectively) (Table 1).

Table 1. Demographic variables, the mean of FEV1 measurement, FEV1 percent, FEV1/FVC percent and CAT questionnaire score before study.

\begin{tabular}{|c|c|c|c|c|c|}
\hline \multicolumn{2}{|l|}{ Variable } & Control group; $n=23$ & Intervention group; $n=23$ & Total; $\mathrm{n}=46$ & p-value \\
\hline \multicolumn{2}{|c|}{ Age $($ Mean \pm SD; year $)$} & $41.00 \pm 6.94$ & $44.21 \pm 5.93$ & $42.60 \pm 6.59$ & 0.098 \\
\hline \multirow[t]{2}{*}{ Gender $(\mathrm{n}, \%)$} & Male & $10,21.7$ & $10,21.7$ & $20,43.5$ & \multirow[t]{2}{*}{1.000} \\
\hline & Female & $13,28.3$ & $13,28.3$ & $26,56.5$ & \\
\hline \multicolumn{2}{|c|}{ FEV1 (Mean \pm SD; liter) } & $2.62 \pm 0.67$ & $2.43 \pm 0.94$ & $2.53 \pm 0.81$ & 0.440 \\
\hline \multicolumn{2}{|c|}{ FEV1 (Mean \pm SD; \%) } & $82.81 \pm 16.09$ & $75.50 \pm 22.16$ & $79.15 \pm 19.50$ & 0.207 \\
\hline \multicolumn{2}{|c|}{ FEV1/FVC $($ Mean \pm SD; \%) } & $78.70 \pm 8.23$ & $73.79 \pm 11.23$ & $76.25 \pm 10.04$ & 0.098 \\
\hline \multicolumn{2}{|c|}{ CAT questionnaire (Mean $\pm \mathrm{SD} ; \mathrm{n})$} & $23.21 \pm 3.27$ & $24.26 \pm 4.37$ & $23.73 \pm 3.85$ & 0.365 \\
\hline
\end{tabular}


We also evaluated the mean of FEV1 percent, FEV1 measurement and CAT questionnaire score differences before and after treatment between the two groups as shown in Table 2. Results showed that the mean of FEV1 percent and CAT questionnaire score difference were significant in the intervention group unlike the control group ( $\mathrm{p}=0.001$, $\mathrm{p}<0.001$ respectively) but the mean of FEV1 measurement between the intervention and the control group was not significant $(\mathrm{p}=0.50)$ (Table 3$)$.

Table 2. The mean of FEV1 measurement, FEV1 percent, FEV1/FVC percent and CAT questionnaire score changes in both groups, before and after three months treatment

\begin{tabular}{|l|l|l|l|}
\hline Group & Variable & Mean \pm SD & p-value \\
\hline Intervention & FEV1 percent & $-17.72 \pm 13.47$ & $<0.001$ \\
\cline { 2 - 4 } & FEV1 measurement & $-0.34 \pm 0.45$ & 0.002 \\
\cline { 2 - 4 } & FEV1/FVC percent & $-5.46 \pm 9.45$ & 0.011 \\
\cline { 2 - 4 } & CAT questionnaire & $19.47 \pm 4.84$ & $<0.001$ \\
\hline \multirow{5}{*}{ Control } & FEV1 percent & $-6.06 \pm 7.63$ & 0.001 \\
\cline { 2 - 4 } & FEV1 measurement & $-0.24 \pm 0.55$ & 0.049 \\
\cline { 2 - 4 } & FEV1/FVC percent & $-2.08 \pm 5.30$ & 0.073 \\
\cline { 2 - 4 } & CAT questionnaire & $12.52 \pm 4.91$ & $<0.001$ \\
\hline
\end{tabular}

Table 3. Comparison of the mean difference of FEV1 measurement, FEV1 percent and CAT questionnaire score between the two groups.

\begin{tabular}{|l|l|}
\hline Variable (differences between two groups) & $\mathrm{p}$-value \\
\hline FEV1 percent difference & 0.001 \\
\hline FEV1 measurement difference & 0.50 \\
\hline CAT questionnaire difference & $<0.001$ \\
\hline
\end{tabular}

\section{Discussion}

To date, there has not been a human study in this field and this is the first clinical trial on evaluation of the effect of camel milk in patients with asthma. The results of this trial showed that camel milk with standard medicinal therapy was helpful in asthma treatment by improving asthma symptoms and spirometric indices. In this study, changes in the mean of FEV1 measurement and FEV1 percent in the intervention and control groups were significant after the treatment but the intervention group showed more improvement compared to the control group. Also FEV1/FVC percent in the intervention group, unlike the control group, was significant.

Considering the high importance of FEV1 percentage and CAT questionnaire in following up the patients, the differences between these variables were also investigated in the intervention and control groups. In this study, the values of FEV1 were evaluated in two variable types: FEV1 measurement and FEV1 percent. Of course, the percentage of FEV1 had a higher accuracy in treatment follow up in asthmatic patients. Generally, pulmonary function tests are not necessarily consistent with the clinical symptoms of patients in adults and children. On the other hand, spirometry of patients with asthma may be normal due to low physical activity or absence of asthma attacks. The evaluation of the FVC variable is not important in following up the response to asthma treatment, as well as the ratio of FEV1 to FVC in general. FEV1/FVC percentages indicate the blockage and are not important in response to treatment. In addition, reduced FEV1 values can be due to poorly performing spirometry.

For this purpose, FEV1 is used to check the changes in the severity of asthma, and to track the severity of the disease, after the initial diagnosis of asthma is confirmed. The percentage of FEV1 is a very important factor, which is also related to the diameter of the airways. At the same time, according to a study by Klich et al., despite the importance of high FEV1 in post-treatment follow-up, the measurement of this variable alone increases the risk of asthma attacks. So there is a need for a questionnaire based on the symptoms of the patients to resolve this problem (22). A review study by Ravaghi et al. (23) in 2018 on herbal and animal-derived remedies for obstructive pulmonary disease, reported that animal resources, such as camel's milk and its various products, have comprehensively been dealt with regarding their nutritive and therapeutic effects, but in spite of various experimental and animal studies, lack of comprehensive clinical trials focusing on the regarded effects remain to reconfirm traditional knowledge. But in the present study, we have found that camel milk, with the standard asthma treatment, would be very helpful, and has somewhat proved the effect of this nutrient in the treatment of asthma. In a study conducted at the Mashhad Pharmaceutical Faculty to investigate the antihistaminic effect of camel milk on 
tracheal tissues isolated from guinea pigs for bronchodilatory effects of camel milk, the results showed that camel milk had no certain antihistamine effects on the trachea of guinea pigs. Therefore, it seems that it has bronchodilatory work through other mechanisms (20). Also, the bronchodilatory effect of camel milk was significant and this effect increased by increasing duration of contact with camel milk, while the effect was not observed in placebo (5) .In a study by Arab $\mathrm{H}$ et al., of mice that suffer from colitis, camel milk decreased the levels of TNF- $\alpha$ cytokines. On the other hand, it decreased the leukocytes influx into colon tissues and decrease oxidative stress (24). In another study by ZHU W.W et al., it was stated that camel milk in mice with acute respiratory distress syndrome (ARDS), led to decrease in TNF- $\alpha$ and IL-1B and oxidative stress markers (with anti-inflammatory and anti-oxidant properties) (25). In a 2015 study by Ebaid H et al., camel milk peptide (CMP) had a strong antioxidant potential that reduced the effect of free oxygen radicals, so it is useful in wound healing (26). As stated, numerous articles have pointed to anti-inflammatory, anti-oxidant and immunomodulatory effects of camel milk and its proteins in the reduction of the inflammatory cytokines such as TNF-a, VEGF, IL-17, IL-6, IL-1B and TGF-B1 in diverse inflammatory diseases in human and animal samples (17-19, 27-29). In our study, camel milk had positive therapeutic effects on spirometry indices (FEV1/FVC percent, FEV1 measurement and FEV1 percent), CAT questionnaire score and symptoms in asthmatic patients. Likely, it can be reasonable because of the antiinflammatory, antioxidant and immunomodulatory effects of camel milk. As stated above, asthma is an inflammatory disease in which inflammation plays a key role. Cytokines, including interleukin (IL-4, IL-5, IL-9 and IL -13), pro-inflammatory cytokines such as tumor necrosis factor-a (TNF-a) and IL-1b, chemokines, eotaxin and monocyte chemotactic protein (MCP-1), and growth factors, such as tissue growth factor-b (TGF-B) and epidermal growth factor are increased expression mediators (30). On the other hand, it is specified that reactive oxygen species also play a key role in airways inflammation and asthma severity determination (31). Possibly, the beneficial effects of using camel milk in the treatment of asthmatic patients is due to the presence of these anti-inflammatory and antioxidant components, which requires accurate studies of this issue. We aimed to investigate the effect of camel milk with standard asthma treatment on the symptom healing of these patients. In this study, 23 asthmatic patients with study conditions entered each group.

\section{Limitations}

One of the limitations of this study was the lack of co-operation of patients with spirometry and their follow-up, which attempts were made to address the limitation with further explanation and follow up by the researchers. The late referral of patients and the time limit for carrying out the study were also other study limitations. In addition, blinding was not done in this study.

\section{Conclusions}

In this study, we found that camel milk, with the standard asthma treatment, would be very helpful, and somewhat proved the effect of this nutrient in the treatment of asthma. Accordingly, camel milk can be used as an adjunct treatment to improve asthmatic patients and possibly reduce the dosage of drugs used in asthma. More extensive research is needed on the use of camel milk on various types of asthma, and the identification of its exact mechanism of action can be a good basis for future research in this area.

\section{Acknowledgments:}

This article is the result of the support of the Research Vice-Chancellor of Mashhad University of Medical Sciences from the Ph.D. Student's Thesis, No. 941287, which is hereby appreciated. The authors have no conflicts of interest.

Funding: This study was supported by Mashhad University of Medical Sciences Research Council, Mashhad, Iran (Ref: 941287). The authors have no conflicts of interest.

Clinical Trial Registration: This study was registered at the Iranian Registry of Clinical Trials (http://www.irct.ir) with the registration code IRCT2016102930541N1.

\section{Conflict of Interest:}

There is no conflict of interest to be declared.

Authors' contributions:

All authors contributed to this project and article equally. All authors read and approved the final manuscript. 


\section{References:}

1) Kasper LD, Braunwald E, Hauser S. Harison's Principles of internal medicine. 18th Ed. New York: McGraw-Hill; 2011.

2) Varmaghani M, Farzadfar F, Sharifi F, Rashidian A, Moin M, Moradi-Lakeh M, et al. Prevalence of Asthma, COPD, and Chronic Bronchitis in Iran: A Systematic Review and Meta-analysis. Iran J Allergy Asthma Immunol. 2016; 15(2):93-104. PMID: 27090362.

3) Arash M, Shoghi M, Tajvidi M. Assessing effects of asthma on patints' functional status and life. Journal of Urmia Nursing and Midwifery Faculty. 2010; 8(1): 1-5.

4) Sharifi L, Pourpak Z, Fazlollahi MR Bokaie S, Moezzi H R, Kazemnejad A, et al. Asthma Economic Costs in Adult Asthmatic Patients in Tehran, Iran. Iran J Public Health. 2015; 44(9):1212-8. PMID: 26587495. PMCID: PMC4645778.

5) "GINA report," Global Strategy for Asthma Management and Prevention, 2017, http://www.ginasthma.org/.

6) Ye Q, He X-O, D'Urzo A. A Review on the Safety and Efficacy of Inhaled Corticosteroids in the Management of Asthma. Pulm Ther. 2017; 3(1):1-18. DOI: https://doi.org/10.1007/s41030-017-0043-5.

7) World Health Organization. WHO traditional Medicine Strategy: 2014-2023. Geneva: World Health Organization; 2013.

8) Noras MR, Yousefi M, Kiani MA. Complementary and Alternative Medicine (CAM) Use in Pediatric Disease: A Short Review. Int J Pediatr: 2013; 1(2):45-9. DOI: 10.22038/ijp.2013.2259.

9) Hosseini SM, Zibaee S, Yousefi M, Taghipour A, Noras M. Camel's milk: nutrition and health perspectives of Iranian traditional medicine. Int J Pediatr. 2015; 3:593-598. DOI: 10.22038/ijp.2015.4704.

10) Asres A, Yusuf M. Traditional Consumption, Therapeutic Value and Its Derived Dairy Products of Dromedary Camel (Camelus Dromedaries) Milk in Somali Regional State, Eastern Ethiopia; a Review. J Biol Agric Healthc. 2014; 4(25): 140-45.

11) Sazmand A, Joachim A. Parasitic diseases of camels in Iran (1931-2017) - a literature review Maladies parasitaires des dromadaires en Iran (1931-2017) - Revue de la littérature. Parasite. 2017; 24:21. doi: 10.1051/parasite/2017024 PMID: 28617666. PMCID: PMC5479402.

12) Mansour AA, Nassan MA, Saleh OM, Soliman MM. Protective effect of camel milk as anti-diabetic supplement: biochemical, molecular and immunohistochemical study. AJTCAM. 2017; 14(4):108-19. DOI: 10.21010/ajtcam.v14i4.13. PMID: 28638873 PMCID: PMC5471457.

13) Restani P, Gaiaschi A, Plebani A, Beretta B, Cavagni G, Fiocchi HR, et al. 1999. Cross-reactivity between milk proteins from different animal species. Clin Exp Allergy. 29:997-1004. doi: 10.1046/j.13652222.1999.00563.x. PMID: 12487198.

14) Yagil R. Camel milk and its unique anti-diarrheal properties. Isr Med Assoc J. 2013; 15:35-36. 1. PMID: 23484237.

15) Clutton-Brock J. Camels and Llamas. A Natural History of Domesticated Mammals. 2nd ed. Cambridge, England: The Press Syndicate of The University of Cambridge; 1999: 151-159.

16) Makinen-Kiljunen S, Palosuo T. 1992. A sensitive enzyme-linked immunosorbent assay for determination of bovine beta-lactoglobulin in infant feeding formulas and human milk. Allergy. 47:347-352. PMID: 1456405. doi: 10.1111/j.1398-9995.1992.tb02070.x

17) Merin U, Bernstein S, Bloch-Damti N, Yagil R, van Creveld C, Lindner P, Gollop N. 2001. A comparative study of milk proteins in camel (Camelus dromedarius) and bovine colostrum. Livestock Product Sci. 67:297-301. DOI: https://doi.org/10.1016/S0301-6226 (00)00198-6.

18) El-Agamy E, Nawar M, Shamsia SM, Awad S, Haenlein G. 2009. Are camel milk proteins convenient to the nutrition of cow milk allergic children? Small Rumin Res. 82:1-6. 1. DOI: https://doi.org/10.1016/j.smallrumres.2008.12.016.

19) Tamara Mihic, Daniel Rainkie, Kyle John Wilby, Shane Ashley Pawluk. The Therapeutic Effects of Camel Milk: A Systematic Review of Animal and Human Trials. J Evid Based Complementary Altern Med. 2016; 21(4):NP110-26. DOI: 10.1177/2156587216658846. PMID: 27432772.

20) Boskabadi M H, Pour Hosseini A, Dean Mohammadi E, Mahdavi M R, Emamian A. Anti-cholinergic and anti-histaminic study of camel milk and urine on the isolated guinea pig trachea. The Book of Synopses of Thirteenth Iranian Congress of Physiology and Pharmacology, p. 359, 1376.

21) Martin RJ, Szefler SJ, King TS, Kraft M, Boushey HA, Chinchilli VM, et al. The Predicting Response to Inhaled Corticosteroid Efficacy (PRICE) trial. J Allergy Clin Immunol. 2007; 119(1):73-80. DOI: 10.1016/j.jaci.2006.10.035. PMID: 17208587. PMCID: PMC2872157. 
22) Tafuro F, Corradi M, Mutti A. [Interpretative strategies of lung function tests: obstructive pattern]. La Medicina del lavoro.2014; 105(3):197-213. PMID: 25078801.

23) Ravaghi M, Hosseini SM, Salari R, Yousefi M, Herbal and Animal Derived Remedies for Obstructive Pulmonary Disease (Asthma and Chronic Obstructive Pulmonary Disease): A Review Study. J Res Med Dent Sci. 2018; 6 (2):520-526. DOI: 10.5455/jrmds.20186279.

24) Arab HH, Salama SA, Eid AH, Omar HA, Arafa el SA, Maghrabi IA. Camel's milk ameliorates TNBSinduced colitis in rats via downregulation of inflammatory cytokines and oxidative stress. Food Chem Toxicol. 2014; 69:294-302. DOI: 10.1016/j.fct.2014.04.032. PMID: 24788059.

25) Zhu WW, Kong GQ, Ma MM, Li Y, Huang X, Wang LP, et al. Short communication: Camel milk ameliorates inflammatory responses and oxidative stress and downregulates mitogen-activated protein kinase signaling pathways in lipopolysaccharide-induced acute respiratory distress syndrome in rats. J Dairy Sci. 2016; 99(1):53-6. DOI: 10.3168/jds.2015-10005. PMID: 26601576.

26) Ebaid H, Abdel-salam B, Hassan I, Al-Tamimi J, Metwalli A, Alhazza I. Camel milk peptide improves wound healing in diabetic rats by orchestrating the redox status and immune response. Lipids Health Dis. 2015; 14:132. doi: 10.1186/s12944-015-0136-9. PMID: 26498022 PMCID: PMC4619484.

27) Alhaider AA, Abdel Gader AG, Almeshaal N, Saraswati S. Camel milk inhibits inflammatory angiogenesis via downregulation of proangiogenic and proinflammatory cytokines in mice. APMIS. 2014; 122(7):599607. DOI: 10.1111/apm.12199. PMID: 24320686.

28) Soliman MM, Hassan MY, Mostafa SA, Ali HA, Saleh OM. Protective effects of camel milk against pathogenicity induced by Escherichia coli and Staphylococcus aureus in Wistar rats. Mol Med Rep. 2015;12(6):8306-12. DOI: 10.3892/mmr.2015.4486. PMID: 26497900.

29) Mohamed WA, Schaalan MF, El-Abhar HS. Camel Milk: Potential Utility as an Adjunctive Therapy to Peg-IFN/RBV in HCV-4 Infected Patients in Egypt. Nutr Cancer. 2015; 67(8):1305-13. DOI: 10.1080/01635581.2015.1087041. PMID: 26492130.

30) Chung KF, Barnes PJ. Cytokines in asthma. Thorax. 1999; 54: 825-857. DOI: http://dx.doi.org/10.1136/thx.54.9.825.

31) Sahiner UM, Birben E, Erzurum S, Sackesen C, Kalayci O. Oxidative Stress in Asthma. World Allergy Organization Journal. 2011;4(10):151-8. doi: 10.1097/WOX.0b013e318232389e. PMID: 23268432 PMCID: PMC3488912. 